PROCEEDINGS OF THE

AMERICAN MATHEMATICAL SOCIETY

Volume 130, Number 6, Pages 1735-1741

S 0002-9939(01)06285-2

Article electronically published on December 20, 2001

\title{
AN AUTOMATIC ADJOINT THEOREM AND ITS APPLICATIONS
}

\author{
JUNDE WU AND SHIJIE LU
}

(Communicated by N. Tomczak-Jaegermann)

\begin{abstract}
In this paper, we prove the following automatic adjoint theorem: For any sequence spaces $E(X)$ and $F(Y)$, if $E(X)$ has the signed-weak gliding hump property and $A$ is an infinite matrix which transforms $E(X)$ into $F(Y)$, then the transpose matrix $A^{\prime}$ of $A$ transforms $F(Y)^{\beta}$ into $E(X)^{\beta}$, and for any $x \in E(X)$ and $T \in F(Y)^{\beta},[A x, T]=\left[x, A^{\prime} T\right]$. That is, the adjoint operator of $A$ automatically exists and is just the transpose matrix $A^{\prime}$ of $A$. From the theorem we obtain a class of infinite matrix topological algebras $(\lambda, \mu)$, and prove also a $\lambda$-multiplier convergence theorem of Orlicz-Pettis type. The theorem improves substantially the famous Stiles' Orlicz-Pettis theorem.
\end{abstract}

\section{INTRODUCTION}

Let $X, Y$ be Hausdorff topological vector spaces and $L(X, Y)$ the space of all continuous linear operators from $X$ into $Y$. Let $E(X)(F(Y))$ be a vector space of $X$-valued ( $Y$-valued) sequences, if $x \in E(X)$, we denote the $k$ th coordinate of $x$ by $x_{k}$ so $x=\left(x_{k}\right)$. The $\beta$-dual of $E(X)$ (with respect to $Y$ ), denoted by $E(X)^{\beta Y}$, is the space of all sequences $\left(T_{k}\right)=T, T_{k} \in L(X, Y)$, such that the series $\sum_{k=1}^{\infty} T_{k} x_{k}$ converges for each $x \in E(X)$. If $Y$ is the scalar field, we write $E(X)^{\beta Y}=E(X)^{\beta}$. If $x \in E(X)$ and $T \in E(X)^{\beta Y}$, we write $[x, T]=\sum_{k=1}^{\infty} T_{k} x_{k}$.

Let $A_{i j} \in L(X, Y)$ for $i, j \in N$, and let $A$ be the operator-valued matrix [ $\left.A_{i j}\right]$. If for each $x \in E(X)$ and $i \in N$, the series $\sum_{j=1}^{\infty} A_{i j} x_{j}$ converges and the sequence $\left\{\sum_{j=1}^{\infty} A_{i j} x_{j}\right\}_{i=1}^{\infty} \in F(Y)$, then $A$ is said to transform $E(X)$ into $F(Y)$.

The pair $(X, Y)$ is said to have the Banach-Steinhaus property if whenever $T_{k} \in$ $L(X, Y)$ converges pointwise, that is, $\lim _{k} T_{k} x=: T_{0} x$ for each $x \in X$, then the limit operator $T_{0}$ is continuous. In particular, if $X$ is the scalar field $K$, then $(K, Y)$ must have the Banach-Steinhaus property.

We say that a sequence $\left\{z^{(k)}\right\}$, of $X$-valued sequences, is a block sequence if there exists a strictly increasing positive integers sequence $\left\{n_{k}\right\}$ such that

$$
z^{(k)}=\left(0,0, \ldots, 0, z_{n_{k-1}+1}^{(k)}, \ldots, z_{n_{k}}^{(k)}, 0, \ldots\right), \quad \text { where } n_{0}:=0 .
$$

The sequence space $E(X)$ is said to have the signed-weak gliding hump property (Signed-WGHP) if for each $x \in E(X)$ and any block sequence $\left\{x^{(k)}\right\}$ with $x=$

Received by the editors June 27, 2000 and, in revised form, December 7, 2000.

2000 Mathematics Subject Classification. Primary 46A45, 47A05.

Key words and phrases. Sequence space, infinite matrix, adjoint operator.

This research was partially supported by the National Natural Science Foundation of China. 
$\sum_{k=1}^{\infty} x^{(k)}$ (coordinatewise sum), then each subsequence of $\left\{x^{(k)}\right\}$ has a further subsequence $\left\{x^{\left(q_{k}\right)}\right\}$ and a choice of signs $\varepsilon_{k} \in\{-1,1\}$ such that $\bar{x}=\sum_{k=1}^{\infty} \varepsilon_{k} x^{\left(q_{k}\right)} \in$ $E(X)$. The definition of the Signed-WGHP is due to C. Stuart (see [1]).

A sequence space $E(X)$ is said to be a monotone space if $m_{0} E(X)=E(X)$, where $m_{0}$ is the space of scalar sequences with finite range and $m_{0} E(X)$ is the coordinatewise product $(2])$.

Any monotone space $E(X)$ has the Signed-WGHP, while the sequence space $b s=\left\{\left(t_{i}\right): \sup _{n}\left|\sum_{i=1}^{n} t_{i}\right|<\infty\right\}$ has the Signed-WGHP but fails to be monotone (see [3]).

Further, $c_{00}(X)$ denotes the space of $X$-valued sequences which are $\theta$ eventually. If $X$ is the scalar field we write $c_{00}(X)=c_{00}$.

It is well known that for any two dual pairs $\left[X_{1}, X_{1}^{\prime}\right]$ and $\left[Y_{1}, Y_{1}^{\prime}\right]$ and any linear operator $T_{0}: X_{1} \rightarrow Y_{1}$, the adjoint operator $T_{0}^{\prime}: Y_{1}^{\prime} \rightarrow X_{1}^{\prime}$ does not necessarily exist ([4, Lemma 11.1.1]). In this paper, for some sequence spaces and matrix operators, we prove an automatic adjoint theorem. From the theorem, we obtain several new important facts.

\section{The MAIN RESUlts}

In order to prove the automatic adjoint theorem, we at first present a general convergence criterion for double sequences.

Let $(G, P)$ be an abelian quasi-normed group. $s:(G, P) \rightarrow(G, P)$ is said to be an additive isometry if for any $x, y \in G$ we have $s(x+y)=s(x)+s(y)$ and $P(s(x))=P(x)$.

It is clear that the identity mapping and $s(x)=-x$ are both an additive isometry.

Let $p, q \in N .[p, q]=\{k: p \leq k \leq q, k \in N\}$ is said to be an interval of $N$. If $\left\{\Delta_{j}\right\}$ is a sequence of intervals in $N$ with $\max \Delta_{j}<\min \Delta_{j+1}, j \in N$, then $\left\{\Delta_{j}\right\}$ is said to be a strictly increasing sequence of intervals.

Lemma 1. Let $(G, P)$ be an abelian quasi-normed group, for $i, j \in N, x_{i j} \in G$, and the series $\sum_{j=1}^{\infty} x_{i j}, \sum_{i=1}^{\infty} x_{i j}$ and $\sum_{i=1}^{\infty} \sum_{j=1}^{\infty} x_{i j}$ are convergent. If for each strictly increasing sequence of intervals $\left\{\Delta_{n}\right\}$ in $N$ there is a subsequence $\left\{\Delta_{n_{k}}\right\}$ of $\left\{\Delta_{n}\right\}$ and a sequence of additive isometries $s_{k}:(G, P) \rightarrow(G, P)$ such that the series $\sum_{i=1}^{\infty} \sum_{k=1}^{\infty} \sum_{j \in \Delta_{n_{k}}} s_{k}\left(x_{i j}\right)$ is convergent, then the double series $\sum_{i, j} x_{i j}$ also converges and

$$
\sum_{i, j} x_{i j}=\sum_{i=1}^{\infty} \sum_{j=1}^{\infty} x_{i j}=\sum_{j=1}^{\infty} \sum_{i=1}^{\infty} x_{i j} .
$$

Proof. At first, we show that the series $\left\{\sum_{j=1}^{\infty} \sum_{i=1}^{m} x_{i j}\right\}$ converges uniformly with respect to $m \in N$. If not, there exists $\varepsilon_{0}>0$ such that for each $j_{0} \in N$ there exist $j_{k} \geq j_{0}$ and $m_{k}$ satisfying that $P\left(\sum_{j=j_{k}}^{\infty} \sum_{i=1}^{m_{k}} x_{i j}\right) \geq \varepsilon_{0}$. Note that the series $\sum_{j=j_{k}}^{\infty} \sum_{i=1}^{m_{k}} x_{i j}$ is convergent, so there exists $l_{k} \in N$ with $l_{k} \geq j_{k}$ such that $P\left(\sum_{j=l_{k}+1}^{\infty} \sum_{i=1}^{m_{k}} x_{i j}\right)<\frac{\varepsilon_{0}}{2}$. Thus we have

$$
P\left(\sum_{j=j_{k}}^{l_{k}} \sum_{i=1}^{m_{k}} x_{i j}\right) \geq \frac{\varepsilon_{0}}{2} .
$$

Let $j_{0}=1$; it follows from inequality (1) that there exist $m_{1}, j_{1}$ and $l_{1}$ such that $P\left(\sum_{j=j_{1}}^{l_{1}} \sum_{i=1}^{m_{1}} x_{i j}\right) \geq \frac{\varepsilon_{0}}{2}$. Since the series $\sum_{j=1}^{\infty} \sum_{i=1}^{m} x_{i j}$ is convergent, there exists 
$j_{00} \in N$ with $j_{00}>l_{1}$, such that whenever $j_{k} \geq j_{00}$ and $l_{k} \geq j_{00}$, for each $m \leq m_{1}$ we have $P\left(\sum_{j=j_{k}}^{l_{k}} \sum_{i=1}^{m} x_{i j}\right)<\frac{\varepsilon_{0}}{2}$. It follows from (11) that for $j_{00}$, there exist $m_{2}, j_{2} \geq j_{00}$ and $l_{2}$ such that

$$
P\left(\sum_{j=j_{2}}^{l_{2}} \sum_{i=1}^{m_{2}} x_{i j}\right) \geq \frac{\varepsilon_{0}}{2} .
$$

That $m_{2}>m_{1}$ and $j_{2}>l_{1}$ is obvious. Continuing this process we can obtain two increasing sequences of positive integers $j_{1} \leq l_{1}<j_{2} \leq l_{2}<\cdots$ and $m_{1}<m_{2}<$ $m_{3}<\cdots$ such that

$$
P\left(\sum_{j=j_{n}}^{l_{n}} \sum_{i=1}^{m_{n}} x_{i j}\right) \geq \frac{\varepsilon_{0}}{2}, \quad n \in N .
$$

Let $\Delta_{n}=\left\{j: j_{n} \leq j \leq l_{n}, j \in N\right\}$; then $\left\{\Delta_{n}\right\}$ is a strictly increasing sequence of intervals in $N$ and (21) can be written as follows:

$$
P\left(\sum_{i=1}^{m_{n}} \sum_{j \in \Delta_{n}} x_{i j}\right) \geq \frac{\varepsilon_{0}}{2}, \quad n \in N .
$$

Consider the infinite matrix $\left[\sum_{i=1}^{m_{p}} \sum_{j \in \Delta_{n}} x_{i j}\right]_{p n}$. For each $n \in N$, we have

$$
\lim _{p} \sum_{i=1}^{m_{p}} \sum_{j \in \Delta_{n}} x_{i j}=\sum_{i=1}^{\infty} \sum_{j \in \Delta_{n}} x_{i j}
$$

For each strictly increasing positive integers sequence $\left\{n_{q}\right\}$, it follows from the hypothesis that there exist a subsequence $\left\{n_{q_{k}}\right\}$ of $\left\{n_{q}\right\}$ and a sequence of additive isometries $s_{k}:(G, P) \rightarrow(G, P)$ such that the series $\sum_{i=1}^{\infty} \sum_{k=1}^{\infty} \sum_{j \in \Delta_{n_{q_{k}}}} s_{k}\left(x_{i j}\right)$ is convergent, so we have

$$
\lim _{p} \sum_{i=1}^{m_{p}} \sum_{k=1}^{\infty} \sum_{j \in \Delta_{n_{q_{k}}}} s_{k}\left(x_{i j}\right)=\sum_{i=1}^{\infty} \sum_{k=1}^{\infty} \sum_{j \in \Delta_{n_{q_{k}}}} s_{k}\left(x_{i j}\right) .
$$

It follows from [5, Theorem 2.7] that

$$
\lim _{p} \sum_{i=1}^{m_{p}} \sum_{j \in \Delta_{p}} x_{i j}=0 .
$$

This contradicts (3i). So the series $\left\{\sum_{j=1}^{\infty} \sum_{i=1}^{m} x_{i j}\right\}_{m}$ converges uniformly with respect to $m \in N$.

Now, we can prove that the double series $\sum_{i, j} x_{i j}$ converges and

$$
\sum_{i, j} x_{i j}=\sum_{i=1}^{\infty} \sum_{j=1}^{\infty} x_{i j}=\sum_{j=1}^{\infty} \sum_{i=1}^{\infty} x_{i j} .
$$

In fact, for any $\varepsilon>0$, since the series $\left\{\sum_{j=1}^{\infty} \sum_{i=1}^{m} x_{i j}\right\}_{m}$ converges uniformly in $m \in N$, there exists $n_{1} \in N$ such that for each $m \in N$ and $n \geq n_{1}$ we have

$$
P\left(\sum_{j=n+1}^{\infty} \sum_{i=1}^{m} x_{i j}\right)=P\left(\sum_{i=1}^{m} \sum_{j=n+1}^{\infty} x_{i j}\right)=P\left(-\sum_{i=1}^{m} \sum_{j=n+1}^{\infty} x_{i j}\right)<\frac{\varepsilon}{2} .
$$


On the other hand, note that the series $\sum_{i=1}^{\infty} \sum_{j=1}^{\infty} x_{i j}$ is convergent, so there exists $m_{1} \in N$ such that whenever $m \geq m_{1}$ we have

$$
P\left(-\sum_{i=m+1}^{\infty} \sum_{j=1}^{\infty} x_{i j}\right)=P\left(\sum_{i=m+1}^{\infty} \sum_{j=1}^{\infty} x_{i j}\right)<\frac{\varepsilon}{2} .
$$

It follows from (4) and (5) that for $m \geq m_{1}$ and $n \geq n_{1}$, we have

$$
\begin{aligned}
& P\left(\sum_{i=1}^{m} \sum_{j=1}^{n} x_{i j}-\sum_{i=1}^{\infty} \sum_{j=1}^{\infty} x_{i j}\right) \\
& \quad \leq P\left(\sum_{i=1}^{m} \sum_{j=1}^{n} x_{i j}-\sum_{i=1}^{m} \sum_{j=1}^{\infty} x_{i j}\right)+P\left(\sum_{i=1}^{m} \sum_{j=1}^{\infty} x_{i j}-\sum_{i=1}^{\infty} \sum_{j=1}^{\infty} x_{i j}\right) \\
& \quad=P\left(-\sum_{i=1}^{m} \sum_{j=n+1}^{\infty} x_{i j}\right)+P\left(-\sum_{i=m+1}^{\infty} \sum_{j=1}^{\infty} x_{i j}\right) \\
& \quad<\frac{\varepsilon}{2}+\frac{\varepsilon}{2}=\varepsilon .
\end{aligned}
$$

Thus, the double series $\sum_{i, j} x_{i j}$ converges and $\sum_{i, j} x_{i j}=\sum_{i=1}^{\infty} \sum_{j=1}^{\infty} x_{i j}$. Furthermore, it follows from

$$
\begin{aligned}
P\left(\sum_{i=1}^{\infty} \sum_{j=1}^{\infty} x_{i j}-\sum_{j=1}^{n} \sum_{i=1}^{\infty} x_{i j}\right) & =\lim _{m} P\left(\sum_{i=1}^{\infty} \sum_{j=1}^{\infty} x_{i j}-\sum_{j=1}^{n} \sum_{i=1}^{m} x_{i j}\right) \\
& =\lim _{m} P\left(\sum_{i=1}^{\infty} \sum_{j=1}^{\infty} x_{i j}-\sum_{i=1}^{m} \sum_{j=1}^{n} x_{i j}\right)
\end{aligned}
$$

that whenever $n \geq n_{1}$ we have

$$
P\left(\sum_{i=1}^{\infty} \sum_{j=1}^{\infty} x_{i j}-\sum_{j=1}^{n} \sum_{i=1}^{\infty} x_{i j}\right)<\varepsilon .
$$

So,

$$
\sum_{i, j} x_{i j}=\sum_{i=1}^{\infty} \sum_{j=1}^{\infty} x_{i j}=\sum_{j=1}^{\infty} \sum_{i=1}^{\infty} x_{i j}
$$

The lemma is proved.

Let $(G, \tau)$ be a Hausdorff abelian topological group. Then the topology $\tau$ can be generated by a family of quasi-norms (see [6]). Thus, from Lemma 1 we have:

Corollary 1. Let $(G, \tau)$ be a Hausdorff abelian topological group, for $i, j \in N$, $x_{i j} \in G$ and the series $\sum_{j=1}^{\infty} x_{i j}, \sum_{i=1}^{\infty} x_{i j}$ and $\sum_{i=1}^{\infty} \sum_{j=1}^{\infty} x_{i j}$ are convergent. If for each strictly increasing sequence of intervals $\left\{\Delta_{n}\right\}$ in $N$ there exist a subsequence $\left\{\Delta_{n_{k}}\right\}$ of $\left\{\Delta_{n}\right\}$ and a sequence $\left\{\varepsilon_{k}\right\}$ with $\varepsilon_{k}=1$ or $\varepsilon_{k}=-1$ such that the series $\sum_{i=1}^{\infty} \sum_{k=1}^{\infty} \sum_{j \in \Delta_{n_{k}}} \varepsilon_{k} x_{i j}$ is convergent, then the double series $\sum_{i, j} x_{i j}$ is 
also convergent, and we have

$$
\sum_{i, j} x_{i j}=\sum_{i=1}^{\infty} \sum_{j=1}^{\infty} x_{i j}=\sum_{j=1}^{\infty} \sum_{i=1}^{\infty} x_{i j} .
$$

Now, we can prove the following automatic adjoint theorem:

Theorem 1. Let $E(X)$ have the Signed-WGHP and contain $c_{00}(X)$. If $(X, Y)$ has the Banach-Steinhaus property and the matrix $A=\left[A_{i j}\right]$ transforms $E(X)$ into $F(Y)$, then the transpose matrix $A^{\prime}$ must transform $F(Y)^{\beta Y}$ into $E(X)^{\beta Y}$ and for each $x \in E(X)$ and $T \in F(Y)^{\beta Y},[A x, T]=\left[x, A^{\prime} T\right]$, where $A x=$ $\left\{\sum_{k=1}^{\infty} A_{i k} x_{k}\right\}_{i=1}^{\infty}, A^{\prime} T=\left\{\sum_{i=1}^{\infty} T_{i} A_{i j}\right\}_{j=1}^{\infty}$.

Proof. Let $T=\left(T_{i}\right) \in F(Y)^{\beta Y}$, and let $A_{i}$ be the $i$ th row of $A$ so

$$
[A x, T]=\sum_{i=1}^{\infty} T_{i}\left[x, A_{i}\right]=\sum_{i=1}^{\infty} \sum_{j=1}^{\infty} T_{i} A_{i j} x_{j}
$$

for $x \in E(X)$. Thus, for $j \in N$ and $x_{0} \in X$, let $e_{j} \otimes x_{0}$ be the sequence with an $x_{0}$ in the $j$ th coordinate and $\theta$ elsewhere. It follows from $E(X) \supseteq c_{00}$ and since $(X, Y)$ has the Banach-Steinhaus property that, for each $j \in N$, the series $\sum_{i=1}^{\infty} T_{i} A_{i j}$ converges in the strong operator topology of $L(X, Y)$. Since $E(X)$ has the Signed-WGHP, by Corollary 1, if we set $C_{j}=\sum_{i=1}^{\infty} T_{i} A_{i j}$ and $C=\left(C_{j}\right)$, then

$$
[A x, T]=\sum_{i=1}^{\infty} \sum_{j=1}^{\infty} T_{i} A_{i j} x_{j}=\sum_{j=1}^{\infty} \sum_{i=1}^{\infty} T_{i} A_{i j} x_{j}=[x, C]=\left[x, A^{\prime} T\right] .
$$

From (6) we get that $C=\left(C_{j}\right)=A^{\prime} T \in E(X)^{\beta Y}$. Thus the theorem holds.

\section{The topological algebras $(\lambda, \mu)$}

In this section, we present the first application of Theorem 1.

Let $\lambda$ and $\mu$ be scalar-valued sequence spaces and have both the Signed-WGHP and $\lambda \supseteq \mu \supseteq c_{00},(\lambda, \mu)$ be all scalar matrices $A$ such that $A$ transforms $\lambda$ into $\mu$.

It is obvious that $(\lambda, \mu)$ is a vector space under the usually matrix addition and matrix scalar multiplication operations.

If $A=\left(a_{i j}\right)$ and $B=\left(b_{i j}\right) \in(\lambda, \mu)$, it is clear that for each $i \in N,\left\{a_{i j}\right\}_{j=1}^{\infty} \in \lambda^{\beta}$. For each $j \in N$, let $e_{j}$ be the scalar sequence with a 1 in the $j$ th coordinate and 0 elsewhere. It follows from $e_{j} \in \lambda$ and $B e_{j} \in \mu$ that $\left\{b_{i j}\right\}_{i=1}^{\infty} \in \mu$. Note that $\lambda^{\beta} \subseteq \mu^{\beta}$, so for $i, j \in N$, the series $\sum_{k=1}^{\infty} a_{i k} b_{k j}$ is convergent. Thus, we can define the matrix multiplication of $A$ and $B$ by $\left(\sum_{k=1}^{\infty} a_{i k} b_{k j}\right)$.

Theorem 2. $(\lambda, \mu)$ is an algebra. In fact, let $A \in(\lambda, \mu)$, and let $A_{i}$ be the ith row of $A$; then $A_{i} \in \lambda^{\beta} \subseteq \mu^{\beta}$. If $x \in \lambda$, it follows from Theorem 1 and $\lambda^{\beta} \subseteq \mu^{\beta}$ that for each $i \in N,\left[B x, A_{i}\right]=\left[x, B^{\prime} A_{i}\right]$, so we have $(A B) x=A(B x)$. Since $B \in(\lambda, \mu)$, $x \in \lambda$, it follows that $A(B x) \in A(\mu) \subseteq A(\lambda) \subseteq \mu$, i.e., $A B \in(\lambda, \mu)$; thus $(\lambda, \mu)$ is closed with respect to matrix multiplication. Similarly, we can prove that the matrix multiplication is also associative. Thus $(\lambda, \mu)$ is an algebra.

Example 1. Since $b v_{0}$ and $b s$ both have Signed-WGHP, so $\left(b v_{0}, b v_{0}\right)$ and $(b s, b s)$ are both algebras (see [7]). 
Now, we equip $(\lambda, \mu)$ with the strong topology and the weak topology as follows:

The strong topology $T_{s}$ is determined by all the following neighbourhoods $V_{s}(\theta, M, D)$ of $\theta$ in $(\lambda, \mu)$ :

$$
V_{s}(\theta, M, D)=\left\{A \in(\lambda, \mu): \sup _{u \in D, x \in M}|[A x, u]| \leq 1\right\},
$$

here $D$ is a bounded subset of $\left(\mu^{\beta}, \sigma\left(\mu^{\beta}, \mu\right)\right)$ and $M$ is a bounded subset of $\left(\lambda, \sigma\left(\lambda, \lambda^{\beta}\right)\right)$.

The weak topology $T_{w}$ is determined by all the following neighbourhoods $V_{w}(\theta, G, Q)$ of $\theta$ in $(\lambda, \mu)$ :

$$
V_{w}(\theta, G, Q)=\left\{A \in(\lambda, \mu): \sup _{u \in Q, x \in G}|[A x, u]| \leq 1\right\}
$$

here $Q$ is a finite subset of $\mu^{\beta}$ and $G$ is a finite subset of $\lambda$.

It is easy to prove that $\left((\lambda, \mu), T_{s}\right)$ and $\left((\lambda, \mu), T_{w}\right)$ are both locally convex topological algebras (see 8$]$ ). Thus, we have obtained a class of concrete topological algebras $(\lambda, \mu)$.

We will discuss further properties of $(\lambda, \mu)$ in other papers.

\section{On the $\lambda$-MULTiPLier CONVERGENT SERIES}

Now, we present the second application of Theorem 1

In this section, we assume that $\left(E_{0}, \tau\right)$ is a Hausdorff topological vector space with a basis $\left\{b_{i}\right\}$ and coordinate functionals $\left\{f_{i}\right\}, F_{0}=\left\{f_{i}: i \in N\right\} ; \sigma\left(E_{0}, F_{0}\right)$ is the weak topology on $E_{0}$ from the duality between $E_{0}$ and $F_{0}$; and $\lambda \supseteq c_{00}$ is a scalarvalued sequence space. A series $\sum_{j=1}^{\infty} x_{j}$ in $E_{0}$ is said to be $\tau$-subseries convergent if for each subsequence $\left\{x_{n_{j}}\right\}$ of $\left\{x_{j}\right\}$, the series $\sum_{j=1}^{\infty} x_{n_{j}}$ is $\tau$-convergent. A series $\sum_{j=1}^{\infty} x_{j}$ in $E_{0}$ is said to be $\tau-\lambda$-multiplier convergent if for each $\left(t_{j}\right) \in \lambda$, the series $\sum_{j=1}^{\infty} t_{j} x_{j}$ is $\tau$-convergent. The famous Stiles' Orlicz-Pettis theorem shows that (see [9, 10]): Any $\sigma\left(E_{0}, F_{0}\right)$-subseries convergent series must also be $\tau$-subseries convergent, or equivalently, if $\lambda \supseteq c_{00}$ is a monotone space, then any $\sigma\left(E_{0}, F_{0}\right)-\lambda$ multiplier convergent series must also be $\tau-\lambda$-multiplier convergent. Now, we present a general $\lambda$-multiplier convergence theorem.

Theorem 3. If $\lambda$ has the Signed-WGHP and contains $c_{00}$, then any $\sigma\left(E_{0}, F_{0}\right)-\lambda$ multiplier convergent series must also be $\tau-\lambda$-multiplier convergent.

Proof. Let $E=\lambda, F=\left\{(x, x, \ldots): x \in E_{0}\right\}, A=\left(\begin{array}{ccc}x_{1} & x_{2} & \cdots \\ x_{1} & x_{2} & \cdots \\ \vdots & \vdots & \ddots\end{array}\right)$, and $t=\left(t_{j}\right) \in \lambda$, and let $x_{0}=\sum_{j=1}^{\infty} t_{j} x_{j}$ be the $\sigma\left(E_{0}, F_{0}\right)$ sum of this series. Let $K$ be the scalar field. Then $\left(K, E_{0}\right)$ has the Banach-Steinhaus property. For $j \in N$ and $x \in E_{0}$, denote $f_{j} \otimes b_{j}(x)=f_{j}(x) b_{j}$. It follows from Theorem 1 that for each $T \in F^{\beta E_{0}},[A t, T]=$ $\left[t, A^{\prime} T\right]$. On the other hand, it is clear that $\left(f_{i} \otimes b_{i}\right) \in F^{\beta E_{0}}$. Thus,

$$
\begin{aligned}
\sum_{i=1}^{\infty} f_{i}\left(\sum_{j=1}^{\infty} t_{j} x_{j}\right) b_{i} & =\sum_{i=1}^{\infty} f_{i}\left(x_{0}\right) b_{i}=x_{0}=\sum_{j=1}^{\infty} t_{j}\left(\sum_{i=1}^{\infty} f_{i} \otimes b_{i}\left(x_{j}\right)\right) \\
& =\sum_{j=1}^{\infty} t_{j} \sum_{i=1}^{\infty} f_{i}\left(x_{j}\right) b_{i}=\sum_{j=1}^{\infty} t_{j} x_{j},
\end{aligned}
$$

and the theorem is proved. 
Example 2. Each $\sigma\left(E_{0}, F_{0}\right)-b s$-multiplier convergent series must be also $\tau-b s$ multiplier convergent.

Since $b s$ has the Signed-WGHP, but $b s$ is not a monotone space, Theorem 3 substantially improved the Stiles' Orlicz-Pettis theorem.

The authors wish to express their thanks to the referee for his valuable comments and suggestions.

\section{REFERENCES}

1. C. E. Stuart, Weak sequential completeness in sequence spaces, Thesis, New Mexico State University, Las Cruces, NM, 1993.

2. G. Bennet, A new class of sequence spaces with applications in summability theory, J. Reine Angew. Math. 266 (1974), 49-75. MR 49:9585

3. J. Boos and T. Leiger, The signed weak gliding hump property, Acta Comm. Univ. Tartuensis 970 (1994), 13-22. MR 97e:40004

4. A. Wilansky, Modern methods in topological vector spaces, McGraw-Hill, New York, 1978. MR 81d:46001

5. C. Swartz, Infinite matrices and the gliding hump, World Sci. Publ., Singapore, 1996. MR 98b:46002

6. H. Weber, A diagonal theorem. Answer to a question of Antosik, Bull. Polon. Acad. Sci. 41 (1993), 95-102. MR 98c:22001]

7. D. J. H. Garling, The $\beta$ - and $\gamma$-duality of sequence spaces, Proc. Camb. Phil. Soc. 63 (1967), 963-981. MR 36:1965

8. A. Mallios, Topological algebras, selected topics, North-Holland, Amsterdam, 1986. MR 87m:46099

9. N. J. Kalton, The Orlicz-Pettis theorem, Contemp. Math. 2 (1980), 91-100. MR 82g:46027

10. C. Swartz, Subseries convergence in spaces with a Schauder basis, Proc. Amer. Math. Soc. 123 (1995), 455-457. MR 95c:46005

Department of Mathematics, Zhejiang University, Hangzhou, 310027, People's RePUBLIC OF CHINA

E-mail address: wjd@math.zju.edu.cn

Department of Mathematics, Zhejiang University, Hangzhou, 310027, People's RePUBlic OF China 